\title{
Infantile Pompe disease: A case report and review of the Chinese literature
}

\author{
YUN LIU, YANG YANG, BEIBEI WANG, LIZHI WU, HONGLU LIANG, QING KAN, \\ ZHAOLAN CAO, YOUYAN ZHAO and XIAOYU ZHOU
}

Department of Neonates, Nanjing Children's Hospital of Nanjing Medical University, Nanjing, Jiangsu 210008, P.R. China

Received October 28, 2014; Accepted June 16, 2015

DOI: $10.3892 /$ etm.2015.2862

\begin{abstract}
Pompe disease, also known as glycogen storage disease type II, is caused by acid maltase deficiency, and can lead to lysosomal glycogen storage. The primal manifestations may be observed in children and adults, and also in infants. In general, the clinical spectrum in infants is more progressive and lethal than that in older patients. This case report describes the case of a newborn who was found to have cardiac hypertrophy, hepatomegaly and elevated serum enzyme levels, which was characterized by an aspartate aminotransferase level of $95 \mathrm{U} / 1$, lactate dehydrogenase level of $778 \mathrm{U} / 1$ and creatine kinase level of 1,299 U/1. On the basis of the clinical signs and laboratory results, dried blood spots from the baby were tested to determine the acid $\alpha$-glucosidase (GAA) activity, and the result confirmed that the GAA activity was only $0.10 \mathrm{pmol} / \mathrm{punch} / \mathrm{h}$ (normal reference range, 2.88-89.02 $\mathrm{pmol} / \mathrm{punch} / \mathrm{h}$ ) at $\mathrm{pH} 3.8$, which was clearly lower than the normal range, leading to a diagnosis of Pompe disease. Pompe disease is incurable, and before the introduction of enzyme replacement therapy (ERT), pain relief was the main treatment. Recognizing this disease earlier and starting ERT in infants prior to the development of clinical symptoms is likely to improve the quality of life of patients.
\end{abstract}

\section{Introduction}

Pompe disease (OMIM 232300), a rare autosomal recessive disorder, is caused by mutation in the gene encoding acid $\alpha$-1,4-glucosidase (GAA, OMIM 606800), which maps on chromosome 17 , and results in glycogen storage in many tissues. The incidence of Pompe disease appears to vary according to ethnicity. Its estimated frequency is $1 / 50,000$ in

Correspondence to: Professor Xiaoyu Zhou, Department of Neonates, Nanjing Children's Hospital of Nanjing Medical University, 72 Guangzhou Road, Nanjing, Jiangsu 210008, P.R. China

E-mail: xyzhou161@163.com

Key words: Pompe disease, glycogen storage disease typeII, infant, newborn, acid $\alpha$-glucosidase
Taiwan (1), 1/138,000 in the Caucasian population and 1/31,000 in individuals of African ancestry (2). The onset symptoms can appear at any time of life, ranging from 0 to 71 years of age (3). Infantile Pompe disease (IPD), also known as classic Pompe disease, presents with cardiomegaly as the most common symptom. Patients often succumb to cardio-respiratory failure caused by muscle weakness and hypotonia in the first year of life (4). The classic form occurs soon after birth, excluding cardiomyopathy. Patients with this disorder also exhibit muscle weakness, hypotonia, respiratory distress, feeding problems and other symptoms. This study describes a case that was unexpectedly identified, having cardiomegaly and hepatomegaly without progressive motor symptoms or feeding problems, and was diagnosed from the GAA activity following dried blood spot (DBS) testing. IPD has a better response to enzyme replacement therapy (ERT) than non-classic Pompe disease, and the earlier the ERT treatment is initiated, the better the prognosis. The condition of the patient accepting the therapy is considered to be more important than the age of the patient to the outcome (5). ERT was not accepted in the present case because of a poor prognosis and other considerations of her parents.

\section{Case report}

In December 28, 2013, a 12-h-old newborn girl, the first child of non-consanguineous parents was admitted to Nanjing Children's Hospital (Nanjing, China) with a hoarse voice after birth. The baby was born at term with a birth weight of 3,500 g. Following rupture of the membranes $21 \mathrm{~h}$ earlier, the mother delivered the baby at 38 weeks in a delivery room. Prenatal examinations and assays were performed in a timely manner and identified no abnormalities. A general physical examination of the baby was conducted, and the liver edge $\sim 3 \mathrm{~cm}$ below the rib was palpated. No dysmorphic features were identified in the examination. A cardiac ultrasound was ordered to examine the heart, which indicated the presence of cardiac hypertrophy. Serum muscle enzymes, including aspartate aminotransferase, lactate dehydrogenase and creatine kinase were found to be elevated (examination and assay results are listed in Table I). No feeding problems, macroglossia, hypotonia or muscle weakness were exhibited. The blood GAA activity was screened to eliminate Pompe disease. DBSs were sent to Shanghai Institute for Pediatric Research, Xinhua Hospital, 
Table I. Symptoms, examination and assay results of the case.

Symptoms and diagnosis

Assay and examination results

Cardiac hypertrophy

Hepatomegaly

Infantile Pompe disease
AST, 95 U/1; LDH, 778 U/1; CK, 1,299 U/1

Echocardiography: left ventricular postrior wall slightly thickened and ventricular septal thickening; patent ductus arteriosus $2 \mathrm{~mm}$ in diameter; atrial septal defect $3.5 \mathrm{~mm}$ in diameter; no left ventricular outflow tract obstruction

GAA, $0.1 \mathrm{pmol} / \mathrm{punch}^{\mathrm{h}} \mathrm{h}^{\mathrm{a}}$

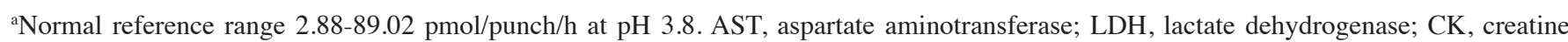
kinase; GAA, acid $\alpha$-glucosidase.

Table II. Signs and symptoms of Pompe disease in reviewed cases (age $<1$ year old).

\begin{tabular}{ll}
\hline Signs and symptoms & No. $(\%)$ \\
\hline Feeding or swallowing problems & $17(51.5)$ \\
$\begin{array}{l}\text { Dysphagia or respiratory problems } \\
\text { Hypotonia and weakness or rapidly } \\
\text { progressive disease course }\end{array}$ & $17(51.5)$ \\
Delayed motor milestones & $21(63.6)$ \\
Cardiomegaly and cardiomyopathy & $19(57.5)$ \\
and/or cardiac failure & $25(75.7)$ \\
Moderate hepatomegaly & $22(66.7)$ \\
Macroglossia & $15(45.4)$ \\
\hline
\end{tabular}

Table III. Laboratory assays and examinations of the reviewed cases (n).

\begin{tabular}{lcc}
\hline Assay/examination & Performed & Abnormal \\
\hline CK & 28 & 26 \\
Transaminasemia & 20 & 20 \\
LDH & 14 & 13 \\
Electromyogram & 7 & 7 \\
Electrocardiogram & 25 & 25 \\
Muscle biopsy & 11 & 10 \\
Cardiac ultrasonography & 23 & 23 \\
GAA activity & 6 & 6 \\
Skin fibroblast & 16 & 16 \\
DBS or leukocyte/lymphocyte & 23 & 23 \\
Genetic analysis & & \\
\hline CK, creatine kinase; LDH, lactate & dehydrogenase; GAA, acid \\
a-glucosidase. & & \\
\hline
\end{tabular}

Shanghai Jiaotong University Medical School (Shanghai, China). A month later, the results were obtained and revealed that the GAA activity was $0.1 \mathrm{pmol} / \mathrm{punch} / \mathrm{h}$ (normal reference range, 2.88-89.02 $\mathrm{pmol} / \mathrm{punch} / \mathrm{h}$ ) at $\mathrm{pH} 3.8$, significantly lower than the normal prescribed minimum, which indicated IPD. Although the family members had no history of this disorder,
Table IV. Mutations in the reviewed cases and whether previously reported or newly identified.

\begin{tabular}{llc}
\hline Status & \multicolumn{1}{c}{ Mutations } & $\begin{array}{c}\text { Chromosome } \\
\text { exon numbers }\end{array}$ \\
\hline Known & p.G689K, p.G576S & $(10,14)$ \\
& p.P266S & $(15,16)$ \\
& p.P361L, p.R437C, p.R600C, & $(16)$ \\
Novel & p.W746S, p.W746* & \\
& p.W738X & $(10,14)$ \\
& p.A702H, p. T711A,p.V723M & $(15)$ \\
& p.R168P, p.E521V,p.R594H & $(16)$ \\
\hline
\end{tabular}

they were advised to undergo genetic testing. When the child was 3.5 months old, telephone follow-up was conducted, and it was found that the child had no feeding problems and was gaining weight. Her muscle tone was normal. Written informed consent was obtained from the patient's parents.

\section{Discussion}

The Wanfang, VIP Chinese Science and Technology Periodicals Database, SinoMed, Duxiu Knowledge Search Database and China National Knowledge Infrastructure databases were searched to identify reports concerning IPD published in Chinese journals. A total of 33 cases from the years 1984 to 2012 were identified, excluding a case from the same hospital. The symptoms of these cases are presented in Table II, and the laboratory and examination results are presented in Table III.

The reviewed cases were not completely investigated by the full range of assays and examinations. In particular, the electromyogram and skin fibroblast testing of GAA activity were infrequently conducted. It is possible that some of the reviewed cases did not report the results of all the tests and examinations that were conducted.

The methods for diagnosing Pompe disease include muscle biopsy, fibroblast GAA testing, gene analysis and peripheral blood GAA activity testing. The GAA assay in muscle or skin fibroblasts remains the gold standard as it can provide a definite diagnosis of Pompe disease (4). In comparison with other methods, peripheral blood GAA activity testing is more 
acceptable because of its feasibility, lack of suffering and for economic reasons. In 2010, Qiu et al reported that DBSs were particularly suitable for the screening of newborns for GAA activity, and that leukocyte GAA activity can also be used in the diagnosis of Pompe disease in newborns and high-risk individuals (6). In 2008, Chien et al confirmed that the early detection of Pompe disease by newborn screening is feasible, by conducting a large-scale newborn screening pilot program in which GAA activity was measured in dried blood spots; the newborn screening resulted in an earlier diagnosis of Pompe disease (at $<1$ month old compared with 3-6 months old in the unscreened control group) (7). In the USA, several pilot programs that are currently ongoing or in the planning stages should provide the data necessary to recommend universal newborn screening for Pompe disease for all infants (8).

In 2005, Kishnani et al reviewed 168 cases of IPD in Israel, Taiwan, North America and Europe, and found that among the cases, $97.6 \%$ were confirmed by GAA activity assay; in only 10 cases $(6.0 \%)$ was GAA mutation analysis pursued (2). In the reviewed cases in mainland China, $33 \%$ of the cases had muscle biopsy, $69.7 \%$ had undergone genetic analysis, $16.2 \%$ underwent skin fibroblast testing of GAA activity, and $48.5 \%$ underwent DBS or leukocyte/lymphocyte testing of GAA activity. The skin fibroblast testing of GAA activity and genetic analysis were mostly reported in Beijing, while the DBS or leukocyte/lymphocyte testing of GAA activity was reported in Shanghai.

Pompe disease is essentially caused by encoding gene mutations that are located in chromosome 17q25.2-q25.3 and contain 19 coding exons. At present, $>200$ different mutations have been reported. The mutations exhibit regional differences; c.-32-13T is the most frequent mutation in late-onset Pompe disease (5), c.1935C >A (p.Asp645Glu) is very frequent in Taiwan (9), and it is also common along the coast of China (10), but there are no reports of the common mutations in mainland China. Only $69.7 \%$ of the reviewed cases had undergone genetic analysis and identified to have mutations; some of the mutations had been reported previously in other countries, and some were new identified (Table IV).

Prenatal examinations are also important for IPD. One of the reviewed cases had been found to have cardiac hypertrophy by routine prenatal ultrasonography at 38 weeks. In 2011, Swarr et al reported a case who had an unusual cardiac 'mass' and biventricular hypertrophy identified by prenatal ultrasound at 30 weeks, which was consistent with the diagnosis of infantile Pompe disease by the detection of GAA activity from a blood spot (11). Following the diagnosis of a patient, it may be necessary for the family to undergo genotype testing and have a genetic consultation with professional geneticists. Zeng et al reported the case of a pregnant woman who already had two baby girls diagnosed with the disease (12). GAA activity was tested in the fetal amniotic fluid and genetic diagnosis was conducted, which confirmed IPD prior to birth (12). If a family has an affected child or sibings, prenatal diagnosis is necessary and it can be confirmed by enzyme analysis of uncultured chorionic villus cells, or by gene analysis through chorionic villus samples or amniocentesis $(4,13)$. Preimplantation genetic diagnosi (PGD), may be available for families in which the disease-causing mutations have been identified (13).
Prior to the emergence of ERT, there was no effective therapy for Pompe disease. No treatment other than supportive care was available to manage the symptoms of patients. In 2006, recombinant human GAA (rhGAA) was approved by the Food and Drug Administration of the USA. The treatment of the disease then changed from symptomatic management to therapeutic intervention. Previous studies reporting the effects of ERT on animals and in clinical trials have demonstrated that ERT has outstanding effects on cardiac hypertrophy and function, and some infants who initiated ERT achieved motor milestones (5). Del Rizzo et al (14) conducted a 26-month follow up of a baby girl who was diagnosed with Pompe disease at the age of 3 days. The child was confirmed to have ventricular hypertrophy by cardiac ultrasound, and ERT was initiated at the age of 20 days. After being treated with ERT for 24 months, the size and function of the child's heart were normal, and growth and motor development were also normal. ERT can greatly modify or moderate the phenotype and progression of the disease $(15,16)$. The effect on muscle function is not as satisfactory as that on the heart because of variability in the skeletal muscle response (16). Early treatment of children with IPD, prior to clinical diagnosis, is of benefit in prolonging survival and improving cardiac and motor function (8), but is not a cure; however, gene therapy may be a therapeutic option (16). The outcome of ERT is likely to be more promising if treatment starts early during the course of the disease. Therefore, the initiation of screening programs for Pompe disease is recommended. Genetic consulting in pregnancy for those with affected children or siblings may be the best method of reducing the incidence or morbidity of the disease.

IPD is fatal and difficult to cure. There maybe no clinical symptoms before the heart, liver or muscles are involved. Early diagnosis is important, as once the patient has respiratory and circulatory failure, there is no cure, although symptomatic treatment may be conducted to alleviate suffering. Following the introduction of ERT, patients are able to have a better quality of life and longer lifetime. However, while ERT only help to slow the progression of the disease, gene therapy may be a promising approach a provide a cure.

\section{References}

1. Lin CY and Shieh JJ: Molecular study on the infantile form of Pompe disease in Chinese in Taiwan. Zhonghua Min Guo Xiao Er Ke Yi Xue Hui Za Zhi 37: 115-121, 1996.

2. Kishnani PS, Hwu WL, Mandel H, Nicolino M, Yong F and Corzo D; Infantile-Onset Pompe Disease Natural History Study Group: A retrospective, multinational, multicenter study on the natural history of infantile-onset Pompe disease. J Pediatr 148: 671-676, 2006.

3. Winkel LP, Hagemans ML, van Doorn PA, Loonen MC, Hop WJ, Reuser AJ and van der Ploeg AT: The natural course of non-classic Pompe's disease; a review of 225 published cases. J Neurol 252: 875-884, 2005.

4. Kishnani PS and Howell RR: Pompe disease in infants and children. J Pediatr 144 (5 Suppl): S35-S43, 2004.

5. van der Ploeg AT and Reuser AJ: Pompe's disease. Lancet 372: 1342-1353, 2008.

6. Qiu WJ, Wang X, Wang Y, Ye J, Han LS, Zhang HW and Gu XF: Establishment and clinical applicafion of dried blood spots and mixed leukocytes for determination of acid alpha-glucosidase activity. Zhonghua Er Ke Za Zhi 48: 55-59, 2010 (In Chinese). 
7. Chien YH, Chiang SC, Zhang XK, Keutzer J, Lee NC, Huang AC, Chen CA, Wu MH, Huang PH, Tsai FJ, et al: Early detection of Pompe disease by newborn screening is feasible: Results from the Taiwan screening program. Pediatrics 122 e39-e45, 2008.

8. Burton BK: Newborn screening for Pompe disease: An update, 2011. Am J Med Genet C Semin Med Genet 160C: 8-12, 2012.

9. Shieh JJ and Lin CY: Frequent mutation in Chinese patients with infantile type of GSD II in Taiwan: Evidence for a founder effect. Hum Mutat 11: 306-312, 1998.

10. Kroos M, Hoogeveen-Westerveld M, van der Ploeg A and Reuser AJ: The genotype-phenotype correlation in Pompe disease. Am J Med Genet Part C Semin Med Genet 160C: 59-68, 2012.

11. Swarr DT, Kaufman B, Fogel MA, Finkel R and Ganesh J: Unusual cardiac 'masses' in newborn with infantile Pompe disease. JIMD Rep 5: 17-20, 2012.
12. Zeng MH, Qiu WJ, Gu XF, Wang Y, Zhou JD, Ye J, Han LS and Zhang HW: Aplication of enzyme assay and gene analysis in the prenatal diagnosis for a family with glycogen storage disease type II. Zhonghua Yi Xue Yi Chuan Xue Za Zhi 28: 261-265, 2011 (In Chinese).

13. Taglia A, Picillo E, D'Ambrosio P, Cecio MR, Viggiano E and Politano L: Genetic counseling in Pompe disease. Acta Myol 30: 179-181, 2011.

14. Del Rizzo M, Fanin M, Cerutti A, Cazzorla C, Milanesi O, Nascimbeni AC, Angelini C, Giordano L, Bordugo A and Burlina AB: Long-term follow-up results in enzyme replacement therapy for Pompe disease: A case report. J Inherit Metab Dis 33 (Suppl 3): S389-S393, 2010.

15. Bailey L: An overview of enzyme replacement therapy for lysosomal storage diseases. Online J Issues Nurs 13, Manuscript 3, 2008.

16. Beck M: Alglucosidase alfa: Long term use in the treatment of patients with Pompe disease. Ther Clin Risk Manag 5: 767-772, 2009. 